\title{
Near-Capacity Turbo Equalization Using Optimized Turbo Codes
}

\author{
Vladimir D. Trajković, Minyue Fu, and Peter J. Schreier \\ School of Electrical Engineering and Computer Science \\ University of Newcastle \\ Callaghan NSW 2308, AUSTRALIA \\ Email: \{Vladimir.Trajkovic, Minyue.Fu, Peter.Schreier\}@ newcastle.edu.au
}

\begin{abstract}
In this paper we analyze a turbo equalization scheme that combines Maximum a Posteriori Probability (MAP) equalization and turbo decoding. Our aim is to optimize the turbo equalizer in order to approach the information capacity limit for channels with severe Inter-Symbol Interference (ISI). For this purpose, we perform an extensive search for turbo codes that give an SNR-BER performance closest to the channel information capacity limit. Our results show that the optimized turbo equalizer can approach the information capacity limit to within $0.7 \mathrm{~dB}$. We also optimize the turbo equalizer in terms of the minimum number of required turbo decoding iterations. Our results show that a turbo decoder within a turbo equalization loop requires only a small number of iterations. Finally, our analysis reveals that when there are turbo codes with similar extrinsic information transfer characteristics, the computational complexity can be reduced by choosing the code with the smallest constraint length with no loss in SNR-BER performance.
\end{abstract}

\section{INTRODUCTION}

Turbo equalization, originally proposed in [1], is an iterative detection technique similar to turbo decoding [2]. The original turbo equalizer [1], [3] combines Maximum Likelihood (ML) or trellis-based equalization and ML channel code decoding in a serially concatenated scheme. In [4], a turbo equalization scheme that employs a turbo code as a channel code was proposed, and later investigated in [5].

Three-stage concatenated turbo systems that employ Serial Concatenated Convolutional Codes (SCCC) have been analyzed in [6]-[8]. In [6] the convergence behavior of three-stage serial systems has been analyzed and capacity approaching performance employing SCCC has been reported, based on the Extrinsic Information Transfer (EXIT) chart [9] analysis only, without Monte Carlo simulation tests.

Lower-complexity turbo equalizers, where ML equalization has been replaced by a low complexity Interference Canceler (IC), were analyzed in [8], [10]-[14]. However, the reduction in the computational complexity is obtained at the expense of degrading the SNR-BER performances of these detectors, which is about $2 \mathrm{~dB}$ for the low complexity schemes of practical importance, as reported in [12].

In this paper we analyze turbo equalization systems that employ MAP equalization and Parallel Concatenated Convolutional (turbo) codes. Our goal in this paper is to find equalizers with performance close to the information capacity

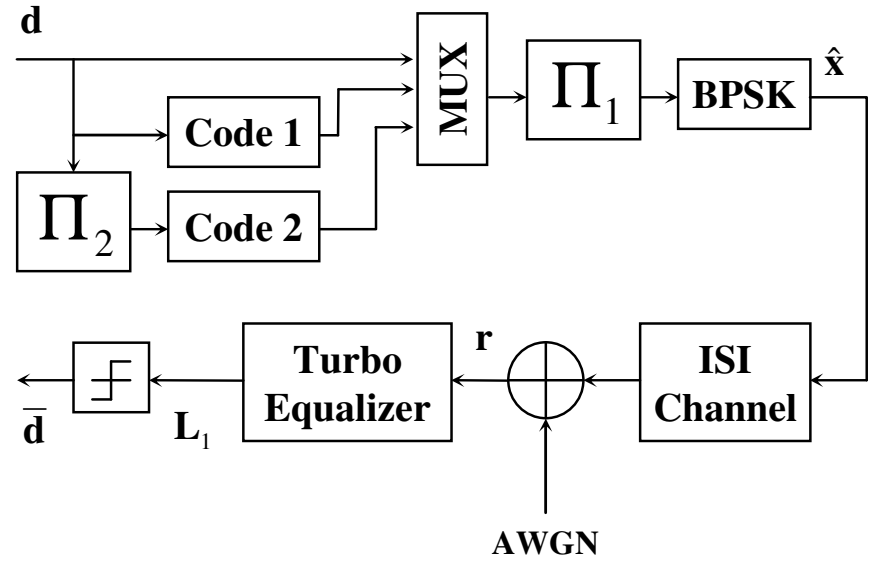

Fig. 1. System model

limit. We do this by employing an extensive search for good constituent codes for turbo coded turbo equalization systems based on EXIT chart analysis. We find that, for certain severe Inter-Symbol Interference (ISI) channels, the optimized turbo equalization scheme can approach the information capacity limit to within $0.7 \mathrm{~dB}$. The optimization is performed over all possible combinations of generator polynomials for constraint lengths up to five.

Our analysis also reveals that the turbo decoder within a turbo equalizer does not require a large number of turbo decoding (inner) iterations. We also show that when there is more than one turbo code with similar extrinsic information transfer characteristics, the code with the smallest constraint length may be used in order to reduce the computational complexity, with no penalty in SNR-BER performance.

We focus on turbo equalization that employs ML equalization to see what performance can be achieved by the turbo coded turbo equalizer. This provides a benchmark for lowercomplexity equalizers, although we do not study them in this paper.

Our work is in contrast to previous work on turbo equalization [8], [10]-[14], that has compared the BER performance with the performance of the coded system on an AWGN channel. This comparison is dependent on the particular channel 
code and does not reveal how close the performance is to the channel capacity. Our simulation results, on the other hand, are compared with the channel capacity of the ISI channel.

In contrast to [6], [8], we regard the turbo coded turbo equalizer as a two-stage system, i.e. a concatenation of the MAP equalizer and the turbo decoder. In this way, we avoid using three-dimensional (3D) EXIT charts [6], [8], i.e. our EXIT charts remain two dimensional.

The remainder of the paper is organized as follows. In Section II we describe the system model, in Section III we search for good turbo codes based on their extrinsic information transfer characteristics. In Section IV we present the simulation results for two different ISI channels and two code rates, while the paper is concluded in Section V.

\section{System MOdEL}

Our system model is shown in Fig. 1. At the transmitter side, the information bits are encoded with a turbo code, where a random interleaver denoted by $\Pi_{2}$ is used for the purpose of turbo coding. Coded bits are interleaved using another interleaver $\Pi_{1}$. Here, we refer to the interleaver $\Pi_{1}$ as outer interleaver and ' $"$ ' will be used to denote a sequence interleaved by $\Pi_{1}$. Interleaver $\Pi_{2}$ is referred to as inner interleaver and ' $\sim$ is used to denote a sequence interleaved by $\Pi_{2}$. Interleaved coded bits are BPSK symbol mapped and the symbols are transmitted through an ISI channel. The received signal at the time instant $k$ is given by

$$
r_{k}=\sum_{i=0}^{M-1} h_{i} \cdot \hat{x}_{k-i}+n_{k}
$$

where $M$ is the length of the channel impulse response, $h_{0}, h_{1}, \ldots, h_{M-1}$ are discrete channel coefficients, $\hat{x}_{k}$ is the $k$ th transmitted coded bit after outer interleaving, and $n_{k}$ is a sample of Additive White Gaussian Noise (AWGN). At the receiver side, the received signal is detected by employing a turbo equalizer. The front end of the turbo equalizer is a Maximum a Posteriori Probability (MAP) equalizer which, at iteration $n$, delivers Log Likelihood Ratios (LLRs) for each transmitted bit as

$$
\hat{L}^{E(n)}\left(\hat{x}_{k}\right)=\log \frac{p\left(\hat{x}_{k}=1 \mid \mathbf{r}, \hat{\mathbf{L}}_{a}^{E(n)}\right)}{p\left(\hat{x}_{k}=-1 \mid \mathbf{r}, \hat{\mathbf{L}}_{a}^{E(n)}\right)} .
$$

where $\hat{\mathbf{L}}_{a}^{E(n)}$ is the equalizer's a priori information at iteration $n$ which is extrinsic information delivered by the turbo decoder at the previous iteration $n-1$, i.e. $\hat{\mathbf{L}}_{a}^{E(n)}=\hat{\mathbf{L}}_{e}^{D(n-1)}$. It is calculated as

$$
\hat{L}_{a}^{E(n)}\left(\hat{x}_{k}\right)=\hat{L}^{D(n-1)}\left(\hat{x}_{k}\right)-\hat{L}_{e}^{E(n-1)}\left(\hat{x}_{k}\right) .
$$

At the first turbo equalization iteration $\hat{L}_{a}^{E(n)}=0$. After a certain number of turbo decoding (inner) iterations, the turbo decoder delivers LLRs for all bits (both systematic and parity check) based on the extrinsic information from the equalizer, which is used as a priori information in the decoder, i.e. $\mathbf{L}_{a}^{D(n)}=\mathbf{L}_{e}^{E(n)}=\mathbf{L}^{E(n)}-\mathbf{L}_{e}^{D(n-1)}$. The stream of $\mathbf{L}_{a}^{D(n)}$ is split into three sub-streams, $\mathbf{L}_{a 1}^{D(n)}$, which contains a priori information regarding systematic bits, $\mathbf{L}_{a 2}^{D(n)}$ - containing $a$ priori information about the parity check bits obtained by the first encoder, and $\mathbf{L}_{a 2}^{D(n)}$ relating to the parity check bits obtained by the second encoder. The first decoder performs decoding using $\mathbf{L}_{a}^{D(n)}$ and $\mathbf{L}_{a 1}^{D(n)}$, while the second decoder uses $\tilde{\mathbf{L}}_{a}^{D(n)}$ interleaved by $\Pi_{2}$ and $\mathbf{L}_{a 3}^{D(n)}$. A MAP decoder within the turbo decoder delivers LLRs regarding both systematic and parity check bits. These LLRs are found as [15]

$$
L\left(x_{k}\right)=\log \frac{\sum_{x_{k}=+1} \alpha_{k-1}\left(s^{\prime}\right) \cdot \gamma_{k}\left(s^{\prime}, s\right) \cdot \beta_{k}\left(s^{\prime}, s\right)}{\sum_{x_{k}=-1} \alpha_{k-1}\left(s^{\prime}\right) \cdot \gamma_{k}\left(s^{\prime}, s\right) \cdot \beta_{k}\left(s^{\prime}, s\right)}
$$

where the summations in the numerator and denominator are over all transitions in the trellis diagram for $x_{k}=+1$ and $x_{k}=$ -1 , respectively. Furthermore, $\alpha_{k}(s)=\sum_{s^{\prime}} \gamma_{k}\left(s^{\prime}, s\right) \cdot \alpha_{k-1}\left(s^{\prime}\right)$, $\beta_{k-1}\left(s^{\prime}\right)=\sum_{s} \gamma_{k}\left(s^{\prime}, s\right) \cdot \alpha_{k-1}(s)$, where the initial values of $\alpha$ and $\beta$ are set to 1 . The metric of a branch in the trellis $\gamma_{k}\left(s^{\prime}, s\right)$, producing the output $u_{1}, u_{2}, \ldots, u_{N}$, is obtained as

$$
\gamma_{k}\left(s^{\prime}, s\right)=\prod_{i=1}^{N} p\left(u_{i}\right)
$$

The probabilities in the product in (5) are calculated as

$$
p\left(u_{i}= \pm 1\right)=\frac{e^{ \pm L_{e, i}}}{1+e^{ \pm L_{e, i}}} \cdot \frac{e^{ \pm L_{a, i}^{D}}}{1+e^{ \pm L_{a, i}^{D}}}
$$

where $L_{e, i}$ is a priori information obtained from the other decoder during turbo decoding iterations, and $L_{a, i}^{D}$ is a priori information obtained from the equalizer. For parity check bits $L_{e, i}$ is always 0 , so that for these bits (6) reduces to

$$
p\left(u_{i}= \pm 1\right)=\frac{1}{2} \cdot \frac{e^{ \pm L_{a, i}^{D}}}{1+e^{ \pm L_{a, i}^{D}}}
$$

The approach described here differs from that in [4], where the deinterleaved extrinsic information from the output of the equalizer is not used directly by the turbo decoder, but a translation from LLR format to equivalent soft channel input is performed first.

After a certain number of inner iterations, the LLRs at the output of the turbo decoder are obtained by combining LLRs from both decoders, i.e. the second decoder delivers LLRs about systematic bits and its parity check bits, denoted $\mathbf{L}_{1}$ and $\mathbf{L}_{3}$ in Fig. 2, while the first decoder delivers LLRs for its parity check bits only, denoted $\mathbf{L}_{2}$ in Fig. 2. These three streams are combined to produce $\mathbf{L}^{D}$ which is then used to obtain extrinsic information $\mathbf{L}_{e}^{D}$. After the final outer iteration, hard decisions about systematic bits only are made as $\overline{\mathbf{d}}=\operatorname{sign}\left(\mathbf{L}_{1}\right)$ in order to retrieve transmitted information.

\section{SeArch For Good Turbo Codes}

An EXIT chart for a turbo equalizer has two curves [12]. The first curve is related to the equalizer and it represents the function $I_{o}^{E}=f_{E}\left(I_{i}^{E}\right)$, where $I_{o}^{E}=I\left(L_{e}^{E} ; X\right)$ and $I_{i}^{E}=I\left(L_{a}^{E} ; X\right)$, 


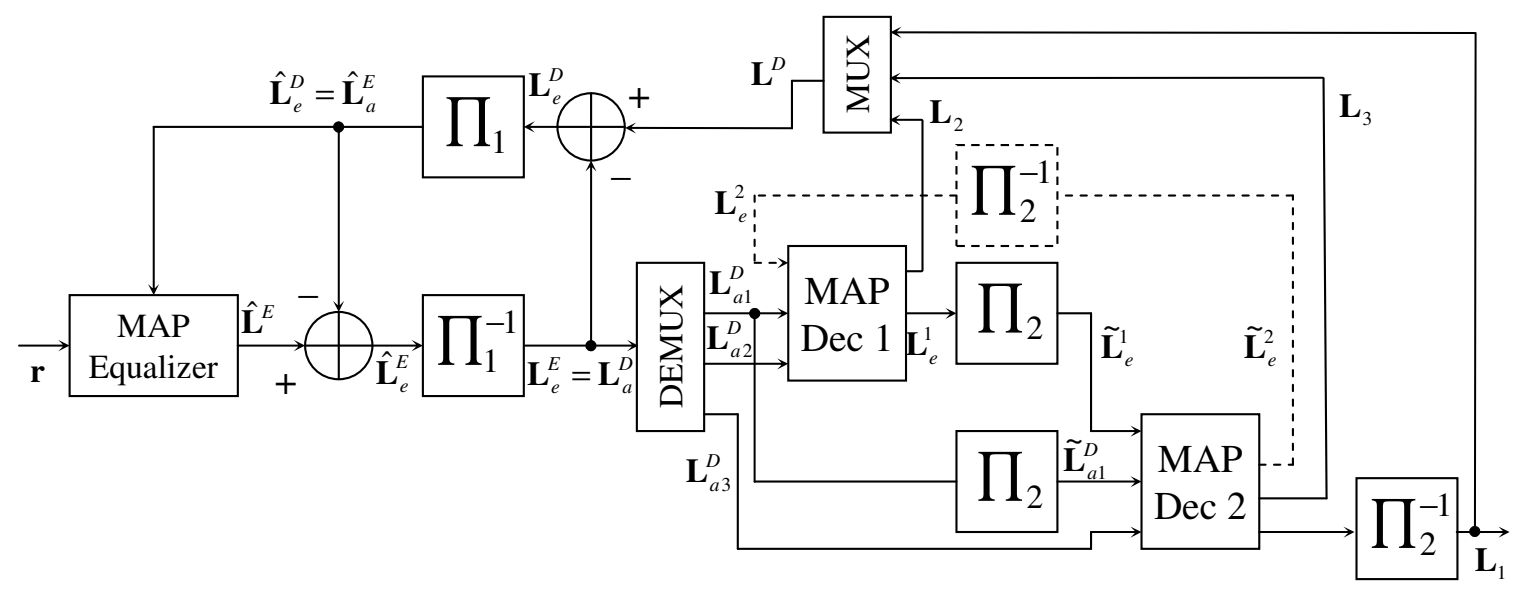

Fig. 2. Turbo equalizer employing turbo decoding

where $I(\cdot ; \cdot)$ denotes mutual information. To find mutual information between $L$-values (a priori or extrinsic information) and transmitted bits, we numerically calculate the following

$$
\begin{array}{r}
I(L ; X)=\frac{1}{2} \sum_{x= \pm 1} \int_{-\infty}^{+\infty} p_{L}(\xi \mid X=x) \times \\
\log _{2} \frac{2 p_{L}(\xi \mid X=x)}{p_{L}(\xi \mid X=-1)+p_{L}(\xi \mid X=+1)} d \xi
\end{array}
$$

where

$$
p_{L}(\xi \mid X=x)=\frac{e^{-\left(\left(\xi-\left(\sigma_{L}^{2} / 2\right) \cdot x\right)^{2} / 2 \sigma_{L}^{2}\right)}}{\sqrt{2 \pi} \sigma_{L}}
$$

In our EXIT chart search for good turbo codes within the turbo equalization loop, we find the function $I_{o}^{D}=f_{D}\left(I_{i}^{D}\right)$ for all combinations of generator polynomials of lengths 2 to 5 , using Monte Carlo simulations. Values $I_{i}^{D}$ and $I_{o}^{D}$ are defined as $I_{i}^{D}=I_{o}^{E}=I\left(L_{e}^{E} ; X\right)$ and $I_{o}^{D}=I_{i}^{E}=I\left(L_{e}^{D} ; X\right)$. We exclude codes with all-zero generator polynomials and codes in which the first coefficient of the feedback polynomial is zero. The approach we use to find the dependence between input and output extrinsic information, i.e. $f_{e}$ and $f_{D}$, is the same as in [9]. Here, we use the assumption that all $L$-values have a Gaussian probability density function. Our focus is on two regions of the EXIT chart: the so-called bottleneck region, which determines the turbo threshold (or 'waterfall' region) of a turbo equalizer and the region where a turbo decoder reaches mutual information very close to $1\left(I_{o}^{D} \approx 1\right)$, which determines the BER floor of the turbo equalizer. To obtain the EXIT charts for different codes, we use $10^{5}$ bits long randomly generated binary sequences, and apply curve fitting. In turbo equalization, the curve related to a turbo decoder is independent on $E_{b} / N_{0}$ because the input into the turbo decoder is extrinsic information from the equalizer, which we assume to be Gaussian distributed. This is important because once determined, turbo decoding curves are fixed and can be used for different ISI channels, SNRs, equalization methods, etc. Only the curve related to the equalizer depends on $E_{b} / N_{0}$. We gradually lower $E_{b} / N_{0}$ to the point where only a small number of turbo decoder curves remain completely below the MAP equalizer curve. Among these curves, we choose the one with the largest bottleneck opening. It is also worthwhile noting that among curves, there is a certain number of almost identical extrinsic information transfer characteristics relating to different turbo codes. This allows a complexity reduction by choosing the code with the shortest constraint length.

An EXIT chart can also be used to optimize turbo equalizers in terms of the number of turbo decoding (inner) iterations. For a particular code, we plot turbo decoder curves for different numbers of iterations, on the same EXIT chart. This method reveals that within the first 2-3 turbo equalization iterations, the turbo decoder requires only a small number of inner iterations and that further iterating does not improve performance. This shows that the computational complexity can be significantly reduced by avoiding unnecessary inner iterations.

\section{Simulation Results}

In this paper we analyze two different ISI channels. The first channel, commonly used in the turbo equalization literature, is $\mathbf{h}_{1}=\left[\begin{array}{lllll}0.227 & 0.46 & 0.688 & 0.46 & 0.227\end{array}\right]^{T}$ from [16]. In this paper we refer to this channel as Channel 1. The EXIT chart describing the turbo equalization of Channel 1, employing a turbo code of code rate $R=1 / 3$ at $E_{b} / N_{0}=2.85 \mathrm{~dB}$, is shown in Fig. 3. We plot EXIT charts for all turbo codes (all combinations of generator polynomials) and eliminate the ones where the equalization curve intersects with a turbo decoding curve. Among the remaining codes, the one with the largest bottleneck opening has generator polynomials $G=\left[\begin{array}{ll}25 & 07\end{array}\right]$, in octal form. We apply an identical analysis for the code rate $R=1 / 2$ and for this scenario we choose the code with generator polynomials $G=\left[\begin{array}{ll}3 & 2\end{array}\right]$.

The second channel is $\mathbf{h}_{2}=\left[\begin{array}{lll}\sqrt{0.45} & \sqrt{0.25} & \sqrt{0.15}\end{array}\right.$ $\sqrt{0.1} \sqrt{0.05}]^{T}$ from [1]. We refer to this channel as Channel 2. For the code rate $R=1 / 3$, we find a number of turbo codes with almost identical extrinsic information transfer characteristics. Among them we choose the one with the smallest constraint length, which has generator polynomials 
$G=\left[\begin{array}{ll}7 & 5\end{array}\right]$. For code rate $R=1 / 2$, we choose $G=\left[\begin{array}{ll}7 & 3\end{array}\right]$. The EXIT chart for this channel at $E_{b} / N_{0}=3 \mathrm{~dB}$, and $R=1 / 2$ is shown in Fig. 4.

Both Figures 3 and 4 also show turbo decoding curves for different numbers of inner iterations. From Fig. 3, we can see that these curves are almost identical for the initial 3 outer iterations. Beyond this point, curves relating to 4 and 10 inner iterations, still follow each other up to $I_{o}^{D} \simeq 0.95$. However, our BER simulations show that an increase in the number of inner iterations from 4 to 10 does not improve the performance significantly, but increases the decoding complexity 2.5 times. Similarly, in Fig. 4, we show turbo decoding curves for 2, 4 and 6 inner iterations. The curves match closely within the first 3-4 outer iterations, so that in this region of the EXIT chart $\left(I_{i}^{E}<0.4\right)$ an increase in the number of inner iterations beyond 2 does not improve the performance. On the other hand, beyond this point 4- and 6-iteration curves show better performance relative to 2 iterations. Our simulation results compare the BER performance of a turbo coded turbo equalizer with the Shannon capacity limit for the two ISI channels. In order to show the information capacity limits for two ISI channels in terms of BER-SNR, we use the algorithm presented in [17] and combine with the approach in [18].

Fig. 5 shows the BER performance of the turbo equalizer employing a turbo code of code rate $R=1 / 3$. The turbo code has been optimized and generator polynomials chosen as described before. The BER performance is compared with the channel capacity limit, which, for Channel 1 , is around $E_{b} / N_{0}=1.43 \mathrm{~dB}$, and for Channel $2, E_{b} / N_{0}=0.9 \mathrm{~dB}$, at low $\operatorname{BER}\left(\leq 10^{-5}\right)$. In each simulation, we generate $2^{18}$ information bits, which after channel coding and modulation gives $3 \cdot 2^{18}$ BPSK symbols. The coded sequence is interleaved using a random interleaver. As mentioned in Section III, our EXIT chart based search for good turbo codes indicated that there is a number of constituent codes with similar BER performance that can approach the capacity limit within $1 \mathrm{~dB}$. For Channel 1, we show the performance of turbo equalizer employing turbo code whose constituent codes have generator polynomials [25 07]. This code requires an $E_{b} / N_{0}$ of $2.3 \mathrm{~dB}$ to achieve a BER of $10^{-5}$, which is only $0.87 \mathrm{~dB}$ away from the capacity limit. The number of outer iterations required to achieve this BER is $n_{\text {out }}=20$. According to Fig. 3, the number of inner iterations is set to $n_{i n}=2$ within the first 3 outer iterations, and $n_{\text {in }}=4$ for outer iterations $n_{\text {out }}=4-20$. For Channel 2 and $\mathrm{G}=\left[\begin{array}{l}5 \\ 5\end{array}\right]$, the turbo equalizer achieves $\mathrm{BER}=10^{-5}$ at about $E_{b} / N_{0}=1.6 \mathrm{~dB}$, which is only $0.7 \mathrm{~dB}$ from the capacity limit. The number of outer iterations for this channel is $14, n_{\text {in }}=2$ within first 3 outer iterations, and $n_{\text {in }}=5$ for outer iterations $n_{\text {out }}=4-14$.

Fig. 6 shows the BER performance of the turbo equalizer where the turbo code has been optimized for the code rate $R=1 / 2$. The results are compared with the ISI channel capacity limit, which, for code rate $R=1 / 2$ and low BER, is around $E_{b} / N_{0}=2.95 \mathrm{~dB}$ for Channel 1 and $2 \mathrm{~dB}$ for Channel 2. Channel coding is employed on $2^{18}$ information bits, which gives total of $2^{19}$ coded bits. The coded bits are

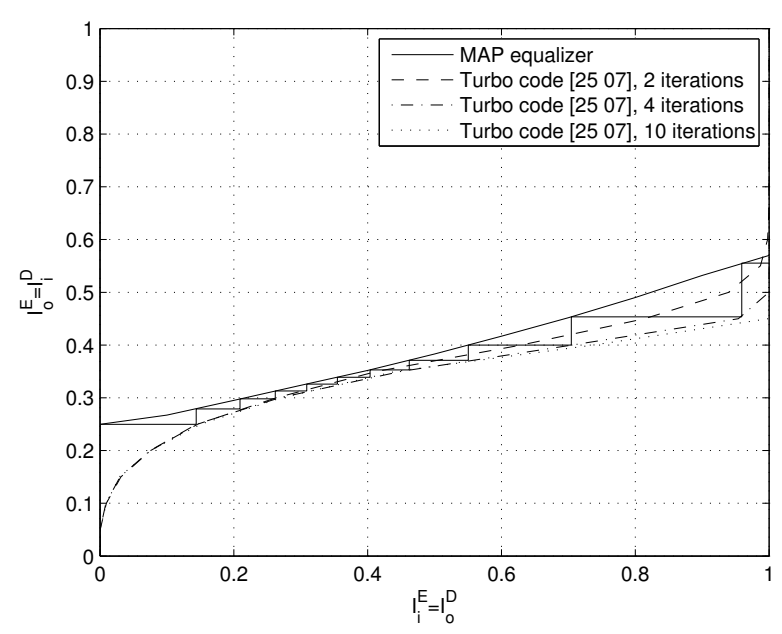

Fig. 3. Turbo equalizer EXIT chart for code rate $R=1 / 3$ and $g 1=25$, $g 2=07$ and $E_{b} / N_{0}=2.85 \mathrm{~dB}$

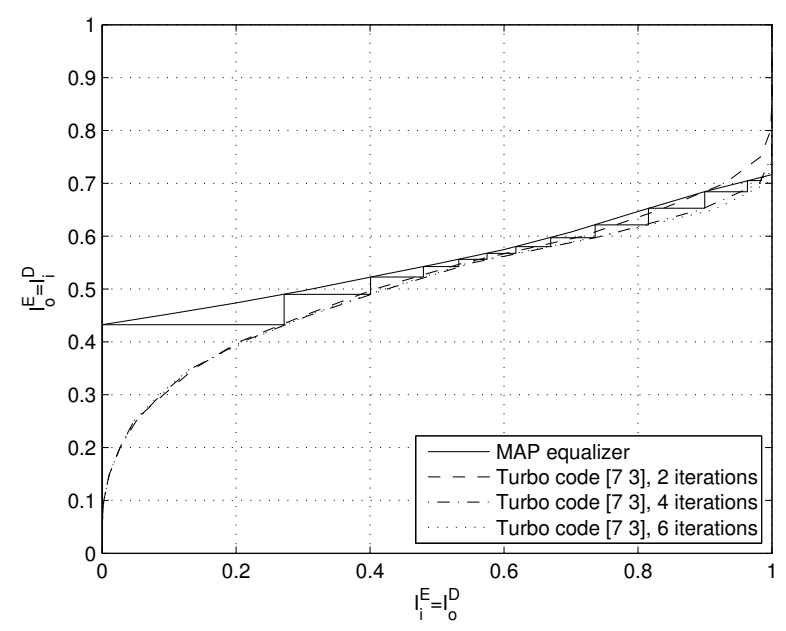

Fig. 4. Turbo equalizer EXIT chart for code rate $R=1 / 2$ and $g 1=7, g 2=3$ and $E_{b} / N_{0}=3 \mathrm{~dB}$

BPSK modulated and interleaved using a random interleaver. A code rate of $1 / 2$ is obtained by alternative puncturing of the parity-check bits as in [2]. Using the same approach as we used for $R=1 / 3$, we find a certain number of turbo codes that approach the capacity within $1 \mathrm{~dB}$. Fig. 6 shows the performance of the turbo equalizer which employs a turbo code whose constituent codes have generator polynomials [3 2]. With this code, the turbo equalizer requires $E_{b} / N_{0}=3.9$ $\mathrm{dB}$ to achieve BER of $10^{-5}$, which is about $0.95 \mathrm{~dB}$ from the capacity limit. The number of outer iterations is $n_{\text {out }}=17$ with $n_{\text {in }}=2$ for $n_{\text {out }}=1-3$, and $n_{\text {in }}=4$ for $n_{\text {out }}=4-17$. For Channel 2 and $G=\left[\begin{array}{ll}7 & 3\end{array}\right]$ the BER lower than $10^{-5}\left(\approx 7 \cdot 10^{-6}\right)$ is achieved at $E_{b} / N_{0}=2.7 \mathrm{~dB}$, or $0.7 \mathrm{~dB}$ from the capacity. For this channel, the number of outer iterations is $n_{\text {out }}=14$ with $n_{\text {in }}=2$ for $n_{\text {out }}=1-3, n_{\text {in }}=4$ for $n_{\text {out }}=4-7$, and $n_{\text {in }}=6$ for $n_{\text {out }}=8-14$. In both Figures 5 and 6 , the information capacity for AWGN and binary signaling is also presented for 


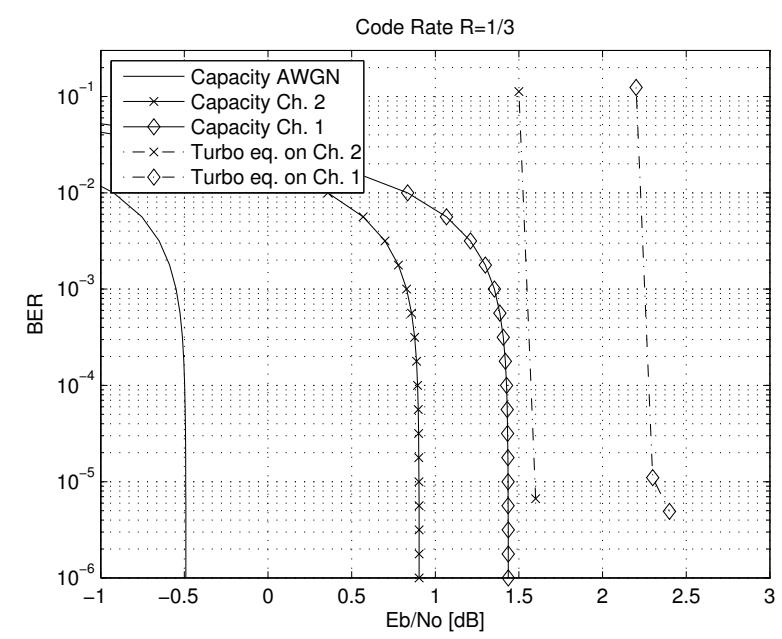

Fig. 5. Turbo equalization BER results for $R=1 / 3$ and two ISI channels.

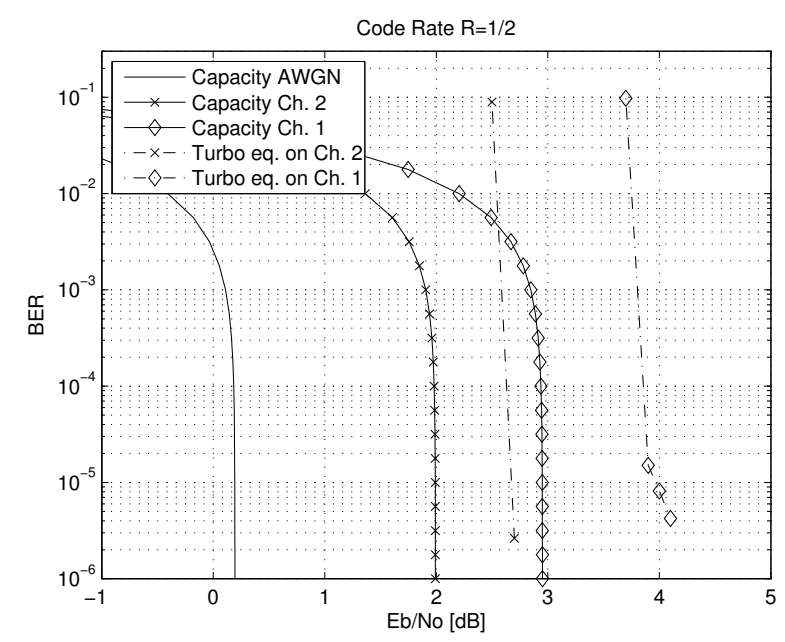

Fig. 6. Turbo equalization BER results for $\mathrm{R}=1 / 2$ and two ISI channels.

the purpose of reference. All BER simulations are performed until 100 erroneous packets have been collected.

\section{CONCLUSION}

In this paper we set out to answer the question whether there exists equalizers that perform close to the capacity limit for severe ISI channels. We were able to answer it in the affirmative by using the EXIT chart analysis to optimize the BER performance of a turbo coded turbo equalization detector. The optimization is performed over all generator polynomials for constituent codes within a turbo encoder, and number of inner (turbo decoding) iterations. We considered turbo codes of rate $\mathrm{R}=1 / 3$ and $\mathrm{R}=1 / 2$, and two different ISI channels. The simulation results show that, for both code rates and channels, we are able to approach the information capacity limit to within $0.7 \mathrm{~dB}$.

\section{REFERENCES}

[1] C. Douillard, M. Jezequel, C. Berrou, A. Piccart, P. Didier, and A. Glavieux, "Iterative correction of intersymbol interference: Turbo equalization," Europ. Trans. Commun., vol. 6, no. 5, pp. 507-511, Sep. 1995.

[2] C. Berrou, A. Glavieux, and P. Thitimajshima, "Near shannon limit errorcorrecting coding: Turbo codes," in Proc. IEEE International Conference on Communications, ICC '93, Geneva, Switzerland, May 1993, pp. 1064-1070.

[3] G. Bauch, H. Khorram, and J. Hagenauer, "Iterative equalization and decoding in mobile communications systems," in Proc. European Personal Mobile Communication Conference, EPMCC '97, Munich, Germany, Sep. 1997, pp. 307-312.

[4] D. Raphaeli and Y. Zarai, "Combined turbo equalization and turbo decoding," in Proc. IEEE Global Communication Conference, GLOBECOM '97, Phoenix, USA, Nov. 1997, pp. 639-643.

[5] W. Ryan, L. McPheters, and S. McLaughlin, "Combined turbo coding and turbo equalization for PR4-equalized lorentzian channels," in Proc. 1998 Conf. on Inf. Sciences and Systems, Princeton University, USA, 1998.

[6] M. Tuchler, "Convergence prediction for iterative decoding of threefold concatenated systems," in Proc. Globecom '02, Taipei, China, Nov. 2002, pp. $1358-1362$.

[7] — , "Design of serially concatenated systems depending on the block length," IEEE Trans. Commun., vol. 52, no. 2, pp. 209-218, Feb. 2004.

[8] J. Wang, S. X. Ng, A. Wolfgang, L. L. Yang, S. Chen, and L. Hanzo, "Near-capacity three-stage mmse turbo equalization using irregular convolutional codes," in Proc. International Symposium on Turbo Codes and Related Topics, Munich, Germany, Apr. 2006.

[9] S. ten Brink, "Convergence behavior of iteratively decoded parallel concatenated codes," IEEE Trans. Commun., vol. 49, no. 10, pp. 17271737, Oct. 2001

[10] A. Glavieux, C. Laot, and J. Labat, "Turbo equalization over a frequency selective channel," in Proc. International Symposium on Turbo Codes, Brest, France, Sep. 1997, pp. 96-102.

[11] C. Laot, A. Glavieux, and J. Labat, "Turbo equalization: Adaptive equalization and channel decoding jointly optimized," IEEE J. Sel. Areas Commun., vol. 9, no. 9, pp. 1744-1752, Sep. 2001.

[12] M. Tuchler, R. Koetter, and A. Singer, "Turbo equalization: Principles and new results," IEEE Trans. Commun., vol. 50, no. 5, pp. 754-767, May 2002.

[13] M. Tuchler, A. Singer, and R. Koetter, "Minimum mean squared error equalization using a priori information," IEEE Trans. Signal Process., vol. 50, no. 3, pp. 673-683, Mar. 2002.

[14] V. D. Trajkovic, P. B. Rapajic, and R. A. Kennedy, "Turbo DFE algorithm with imperfect decision feedback," IEEE Signal Process. Lett., vol. 12 , no. 12 , pp. $820-823$, Dec. 2005.

[15] J. Hagenauer, E. Offer, and L. Papke, "Iterative decoding of binary block and convolutional codes," IEEE Trans. Inf. Theory, vol. 42, no. 2, pp. 429-445, Mar. 1996.

[16] J. Proakis, Digital Communications, 4th ed. New York: McGraw-Hill, 2000.

[17] D. Arnold and H. A. Loeliger, "On the information rate of binaryinput symetric channels," in Proc. IEEE International Conference on Communications, Zurich, Switzerland, 2001, pp. 2692-2695.

[18] B. J. Frey, Graphical Models for Machine Learning and Digital Communication, 1st ed. Cambridge, Massachusetts: The MIT Press, 1998. 